Supporting Information

\title{
The improvement of gas and humidity sensing properties of organ-like MXene by alkaline treatment
}

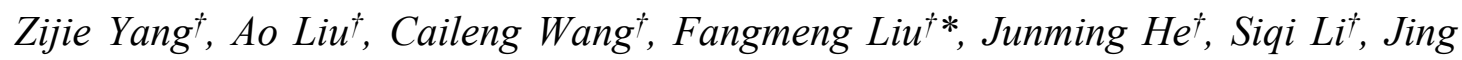
Wang $^{\dagger}$, Rui You*, Xu Yan', Peng Sun', Yu Duan ${ }^{\dagger}$ and Geyu Lü*

$\dagger$ State Key Laboratory on Integrated Optoelectronics, College of Electronic Science and Engineering, Jilin University, 2699 Qianjin Street, Changchun 130012, People's Republic of China.

$\$$ Department of Precision Instrument, Tsinghua University, Beijing 100084, China.

E-mail: liufangmeng@jlu.edu.cn, 1ugy@jlu.edu.cn. 


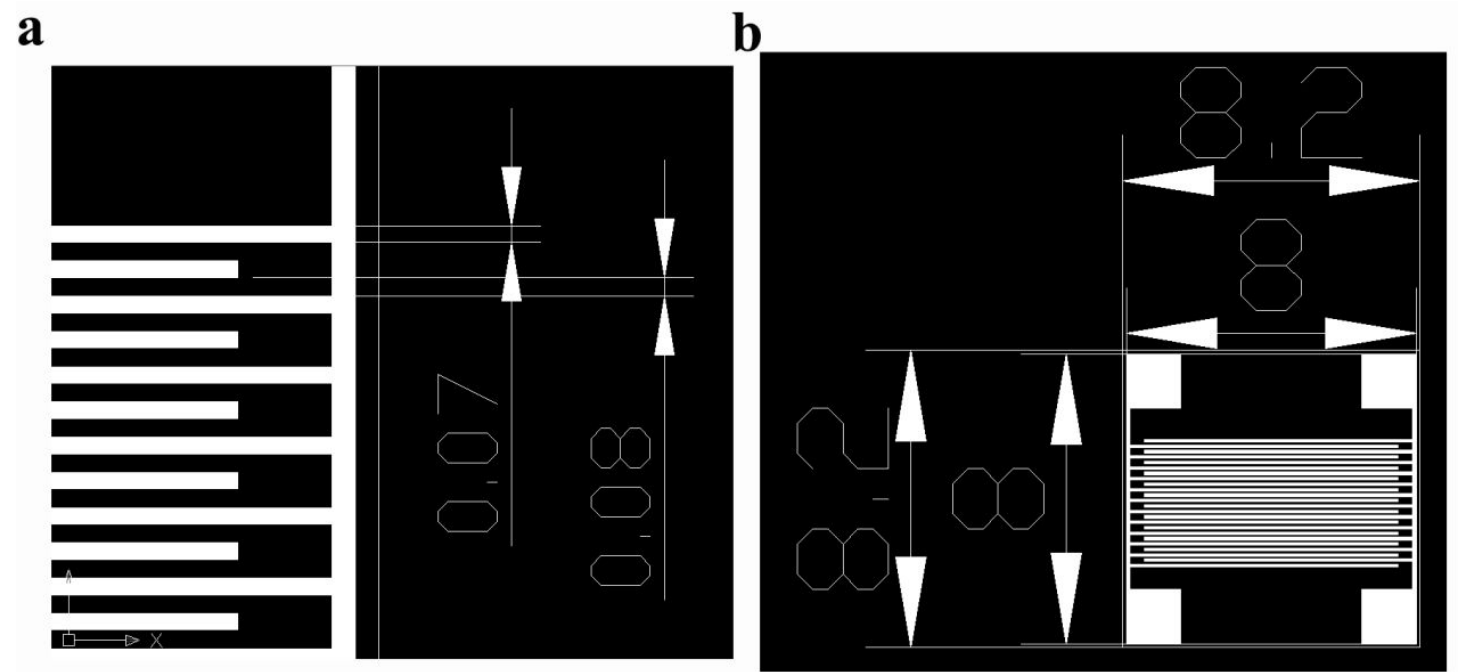

Fig. S1. (a) The local and (b) global parameters schematic of the interdigital electrode. 

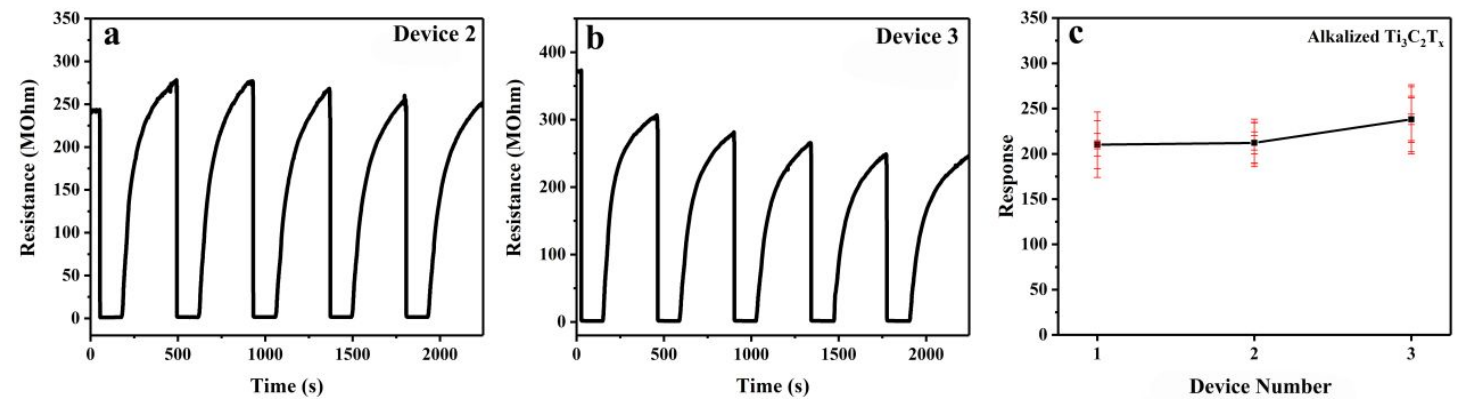

Fig. S2. The repeated response-recovery curve of (a) Device 2 and (b) Device 3 between $11 \% \mathrm{RH}$ and $95 \% \mathrm{RH}$; (c) the error bars of the response.
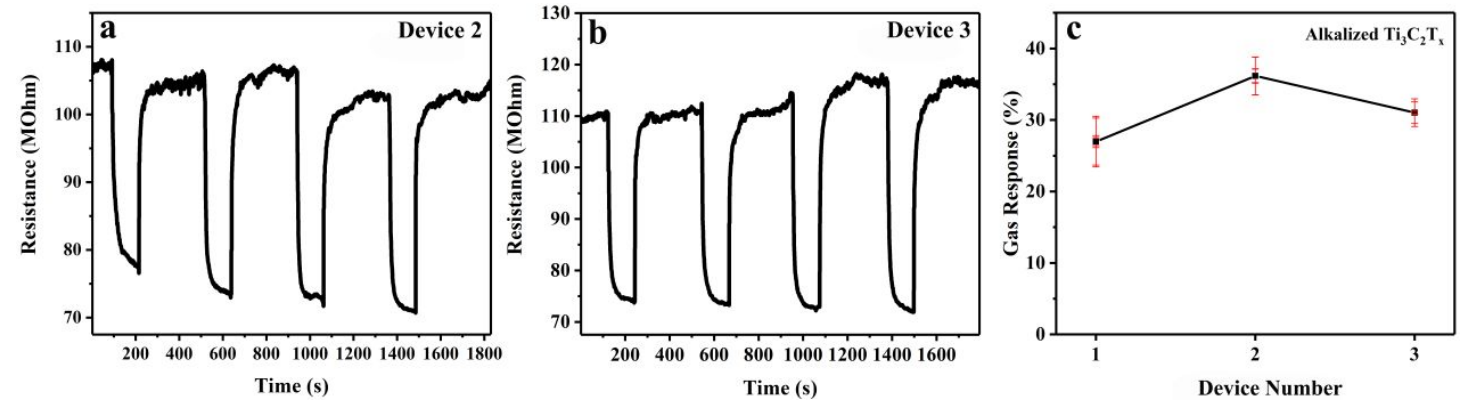

Fig. S3. The repeated response-recovery curve of (a) Device 2 and (b) Device 3 to $100 \mathrm{ppm} \mathrm{NH}_{3}$; (c) the error bars of the response. 